\title{
CONSTANT MEAN CURVATURE SURFACES IN EUCLIDEAN AND MINKOWSKI 3-SPACES
}

\author{
DAVID BRANDER, WAYNE ROSSMAN, AND NICHOLAS SCHMITT
}

\begin{abstract}
Spacelike constant mean curvature surfaces in Minkowski 3 -space $\mathbb{R}^{2,1}$ have an infinite dimensional generalized Weierstrass representation. This is analogous to that given by Dorfmeister, Pedit and $\mathrm{Wu}$ for constant mean curvature surfaces in Euclidean space, replacing the group $S U_{2}$ with $S U_{1,1}$. The non-compactness of the latter group, however, means that the Iwasawa decomposition of the loop group, used to construct the surfaces, is not global. The construction is described here, with an emphasis on the difference from the Euclidean case.
\end{abstract}

Geometry, Integrability and Quantization - Varna 2008

\section{INTRODUCTION}

This article expands on the content of a talk given at the conference Geometry, Integrability and Quantization, in Varna 2008. It discusses the generalized Weierstrass representation for constant mean curvature surfaces in both the Euclidean and in the Minkowski 3-space, with attention given to the difference between these cases. Detailed proofs and further results on the Minkowski case will appear in a forthcoming article by the authors [2].

\section{Constant Mean Curvature Surfaces in Euclidean 3-Space}

2.1. Minimal Surfaces. Constant mean curvature surfaces are mathematical models for soap films and other fluid membranes. A special case is a minimal surface, where the mean curvature is zero. Mathematically, the study of minimal surfaces has been greatly assisted by the well-known Weierstrass representation, which allows one to construct all minimal surfaces from pairs of holomorphic functions via a simple formula. It is based on the fact that the Gauss map of a minimal surface is holomorphic, together with the fact that a CMC surface in general is determined by its Gauss map. Specifically, the Weierstrass representation for minimal surfaces says that, if $g$ is

2000 Mathematics Subject Classification. Primary 53C42, 14E20; Secondary 53A10, $53 \mathrm{~A} 35$.

Key words and phrases. differential geometry, surface theory, loop groups, integrable systems. 
meromorphic, $f$ is holomorphic and $f g^{2}$ is holomorphic, then

$$
\left[\begin{array}{l}
x_{1} \\
x_{2} \\
x_{3}
\end{array}\right]=\left[\begin{array}{c}
\mathfrak{R e} \int_{0}^{z} \frac{f(w)\left(1-g(w)^{2}\right)}{2} \mathrm{~d} w \\
\mathfrak{R e} \int_{0}^{z} \frac{i f(w)\left(1+g(w)^{2}\right)}{2} \mathrm{~d} w \\
\mathfrak{R e} \int_{0}^{z} f(w) g(w) \mathrm{d} w
\end{array}\right]
$$

is a minimal surface in Euclidean 3-space. Conversely, all minimal surfaces are given this way.

2.2. Non-Minimal CMC Surfaces. For non-minimal CMC surfaces, it is no longer true that the Gauss map is holomorphic. It is however, harmonic, and harmonic maps into a symmetric space $G / K$ have a loop group representation; that is, they are represented by certain maps into $\Omega G$, the group of based loops (maps from the unit circle into $G$ which map 1 to the identity element). Now $\Omega G$ admits a complex structure, and the maps in question are holomorphic with respect to this structure. This underlies the generalized Weierstrass representation for CMC surfaces, which was given by Dorfmeister, Pedit and Wu [3]. The practical difference between this representation and that of minimal surfaces described above, is that one needs to perform a loop group decomposition (the Iwasawa decomposition) on the holomorphic data before one can obtain the surface from a simple formula. The Iwasawa decomposition can, in general, be carried out quickly and to arbitrary precision, using numerical methods.

2.3. The DPW Method. The method of Dorfmeister-Pedit-Wu (DPW), gives a holomorphic representation for general harmonic maps from a Riemann surface into a compact symmetric space. In essence, it is based on a more general simple principle, which was also used by Krichever [4], to produce solutions of the sine-Gordon equation from pairs of arbitrary curves. The basic idea, which will be described here, was studied in general in [1], and could be called the KDPW method, after Krichever-DPW.

If $G^{\mathbb{C}}$ is a complex semisimple Lie group, $\Lambda G^{\mathbb{C}}$ denotes the group of maps $\gamma: \mathbb{S}^{1} \rightarrow G^{\mathbb{C}}$, which are of an appropriate smoothness class. A smooth map $F: M \rightarrow \Lambda G^{\mathbb{C}}$, can be thought of as a 1-parameter family of maps, $F_{\lambda}$ : $M \rightarrow G^{\mathbb{C}}$, where $\lambda \in \mathbb{S}^{1}$. A fundamental object in the study of submanifolds of symmetric spaces, is the Maurer-Cartan form, $F_{\lambda}^{-1} \mathrm{~d} F_{\lambda}$, which takes values in the Lie algebra $\mathfrak{g}^{\mathbb{C}}$. If $a$ and $b$ are (extended) integers, say that $F$ is of connection order $(a, b)$ if the Maurer-Cartan form has dependence on $\lambda$ which is a Laurent polynomial as follows:

$$
F_{\lambda}^{-1} \mathrm{~d} F_{\lambda}=\sum_{a}^{b} a_{i} \lambda^{i} .
$$

Basic Principle: The KDPW Method constructs all connection order $(a, b)$ maps, $a<0<b$, from pairs of $(a,-1)$ and $(1, b)$ maps. 
Where this principle has been used, it simplifies the data: for the case of the sine-Gordon equation, the $(a,-1)$ and $(1, b)$ maps are just arbitrary functions of one variable. For the case of harmonic maps, there is just an $(a,-1)$ map (the other one is related to it by an involution), and this is an essentially arbitrary holomorphic map.

To describe one direction of the procedure, we need the Birkhoff decomposition [5], which says that

$$
\mathcal{B}^{ \pm}:=\Lambda^{ \pm} G^{\mathbb{C}} \cdot \Lambda^{\mp} G^{\mathbb{C}},
$$

is open and dense in the identity component of $\Lambda G^{\mathbb{C}}$. Here $\Lambda^{ \pm} G^{\mathbb{C}}$ consists of loops which extend holomorphically to the unit disc $\mathbb{D}$ and its complement $\hat{\mathbb{C}} \backslash \mathbb{D}$ respectively. Thus loops in $\gamma \in \mathcal{B}^{ \pm}$can be factorized $\gamma=\gamma_{ \pm} \gamma_{\mp}$, where $\gamma_{ \pm}$have power series expansions in $\lambda^{ \pm 1}$.

Assume now that $F$ takes values in the open set $\mathcal{B}^{ \pm} \cap \mathcal{B}^{\mp}$. If $F$ is of order $(a, b), a<0<b$, decompose

$$
F=F_{+} G_{-}=F_{-} G_{+} .
$$

Then $F_{+}$is of order $(1, b)$ and $F_{-}$is of order $(a,-1)$. We check this for $F_{+}$:

$$
\begin{aligned}
F_{+}^{-1} \mathrm{~d} F_{+} & =G_{-}\left(F^{-1} \mathrm{~d} F\right) G_{-}^{-1}+G_{-} \mathrm{d} G_{-}^{-1} \\
& =G_{-}\left(\sum_{a}^{b} a_{i} \lambda^{i}\right) G_{-}^{-1}+G_{-} \mathrm{d} G_{-}^{-1} \\
& =c_{0}+\ldots+c_{b} \lambda^{b} .
\end{aligned}
$$

We used the power series expansions of the group elements to deduce the final line. It is possible to normalize the Birkhoff decomposition so that $c_{0}$ is zero, and then $F_{+}$is of order $(1, b)$.

Conversely, given order $(1, b)$ and $(a,-1)$ maps, $F_{+}$and $F_{-}$, we can construct an order $(a, b)$ map $F$. To show the converse, there are two cases, as discussed in [1], and the case which is relevant to this article involves an Iwasawa decomposition, which will be mentioned again below.

After a normalization, both directions are unique, and one obtains a correspondence as follows:

$$
\begin{array}{cc}
F \longleftrightarrow\left\{\begin{array}{l}
F_{+} \\
F_{-}
\end{array}\right\} \\
\operatorname{order}(1, b) \\
\operatorname{order}(a,-1)
\end{array}
$$




\subsection{A Loop Group Representation of Harmonic Maps into Com-} pact Symmetric Spaces. Here we outline results of [3]. The ideas which led to the loop group formulation here are due to many people, and references can be found in [2].

Let $G / K$ be a compact symmetric space, $K=G_{\sigma}$, the fixed point subgroup of an involution $\sigma$. On $\Lambda G^{\mathbb{C}}$, define an involution $\hat{\sigma}$ :

$$
(\hat{\sigma} \gamma)(\lambda):=\sigma(\gamma(-\lambda)) \text {. }
$$

Consider the subgroup of $G$-valued loops which are fixed by this involution, $\Lambda G_{\hat{\sigma}} \subset \Lambda G_{\hat{\sigma}}^{\mathbb{C}} \subset \Lambda G^{\mathbb{C}}$. The "twisting" given by taking elements which are fixed by $\hat{\sigma}$ means that information about the symmetric space $G / K$ is encoded in the twisted subgroup $\Lambda G_{\hat{\sigma}}$.

Let $\Omega$ be a simply connected domain in $\mathbb{C}$.

Suppose: $F: \Omega \rightarrow \Lambda G_{\hat{\sigma}}$ is a connection order $(-1,1)$ map.

Apply the DPW correspondence (1): $F \leftrightarrow\left\{F_{+}, F_{-}\right\}$. In this case, it turns out that $F_{+}$is determined by $F_{-}$, so (1) can be simplified to:

$$
F \leftrightarrow F_{-} .
$$

Now fix $\lambda \in S^{1}$ : then $F_{\lambda}: \Omega \rightarrow G$.

Fact: The projection of $F$, to $G / K$, is a harmonic map $\Omega \rightarrow G / K$ if and only if $F_{-}$is holomorphic in $z$ :

$$
\begin{array}{cccc}
\operatorname{order}(-1,1) \quad F & \leftrightarrow & F_{-} \quad \operatorname{order}(-1,-1) \\
\text { frame for harmonic map } & & \text { holomorphic }
\end{array}
$$

2.5. "Weierstrass Representation" for CMC $H \neq 0$ Surfaces. We have just seen that producing the loop group "frames" $F$ for harmonic maps into $G / K$ amounts to producing a holomorphic connection order $(-1,-1)$ map into $\Lambda G^{\mathbb{C}}$. The recipe is roughly as follows:

(1) Given $a(z), b(z)$ arbitrary holomorphic functions, $a$ non-vanishing. The latter condition assures regularity of the surface. Set

$$
\alpha=\left(\begin{array}{cc}
0 & a(z) \\
b(z) & 0
\end{array}\right) \lambda^{-1} \mathrm{~d} z
$$

(2) Since the 1-form depends only on one complex variable, it is automatically integrable, and there exists (on a simply connected domain) a map $F_{-}: \Omega \rightarrow \Lambda G$, such that $\alpha=F_{-}^{-1} \mathrm{~d} F_{-}$. Additionally, $F_{-}$is holomorphic and, by definition, is of connection order $(-1,-1)$.

(3) Apply KDPW correspondence (2) to get $F$, a frame for a harmonic map.

(4) A CMC surface is obtained from $F$ by a simple formula, the SymBobenko formula (see (6) below). 
In fact, all CMC surfaces in $\mathbb{R}^{3}$ are obtained this way.

2.6. The Iwasawa Decomposition. For the $\leftarrow$ direction of the DPW correspondence (1) one needs the Iwasawa splitting

$$
\Lambda G^{\mathbb{C}}=\Lambda G \cdot \Lambda^{+} G^{\mathbb{C}} .
$$

This holds if $G$ is compact. $F$ is obtained from $F_{-}$via an Iwasawa factorization:

$$
F_{-}=F G_{+} .
$$

More generally, for the $\leftarrow$ direction, the holomorphic map $F_{-}$can be of order $(-1, b)$ where $b \geq-1$, so we could have allowed higher order terms in our initial data (3) given in the above recipe for CMC surfaces.

\section{CMC surfaces in Minkowski 3-Space}

Now we consider the case of spacelike CMC surfaces in Minkowski 3Space, $L^{3}$, which is studied in [2]. The construction is analogous to CMC surfaces in $\mathbb{R}^{3}$, replacing the group $S U(2)$ with the non-compact real form $S U(1,1)$.

Main difference: $S U(1,1)$ non-compact implies that the Iwasawa decomposition is not global.

In fact we show that the Iwasawa splitting is defined on an open dense set (the "big cell"), and that the surfaces have singularities when the holomorphic data encounters this boundary.

3.1. The loop group construction. We use the Pauli matrices:

$$
\sigma_{1}:=\left(\begin{array}{cc}
0 & 1 \\
1 & 0
\end{array}\right), \quad \sigma_{2}:=\left(\begin{array}{cc}
0 & -i \\
i & 0
\end{array}\right), \sigma_{3}:=\left(\begin{array}{cc}
1 & 0 \\
0 & -1
\end{array}\right)
$$

Let $G^{\mathbb{C}}$ be the special linear group $S L_{2} \mathbb{C}$, and define the twisted loop group $\Lambda G_{\hat{\sigma}}^{\mathbb{C}}$ as in Section 2.4, where $\sigma=\operatorname{Ad}_{\sigma_{3}}$. Now the real form $S U_{1,1}$ is the fixed point subgroup with respect to the involution

$$
\tau(x)=\operatorname{Ad}_{\sigma_{3}}\left(\overline{x^{t}}\right)^{-1} .
$$

For our application, however, it turns out that it is convenient to set

$$
G:=\left\{x \in S L_{2} \mathbb{C} \mid \tau(x)= \pm x\right\}
$$

and we consider maps into the subgroup

$$
\Lambda G_{\hat{\sigma}}:=\{x \in \Lambda G \mid \hat{\sigma}(x)=x\} \subset \Lambda G^{\mathbb{C}} .
$$

Note that, defining (if $u$ is a scalar function of $\lambda$ ) $\left.u^{*}(\lambda):=\overline{u\left(\bar{\lambda}^{-1}\right.}\right)$, elements of this subgroup are of the form $\left(\begin{array}{cc}a & b \\ b^{*} & a^{*}\end{array}\right)$ or $\left(\begin{array}{cc}a & b \\ -b^{*} & -a^{*}\end{array}\right)$ depending on whether $\tau(x)=x$ or $\tau(x)=-x$ respectively. 
3.1.1. SU $(1,1)$ Iwasawa decomposition. To describe the decomposition precisely, we define the special loops:

$$
\omega_{m}=\left(\begin{array}{cc}
1 & 0 \\
\lambda^{-m} & 1
\end{array}\right), m \text { odd } ; \quad \omega_{m}=\left(\begin{array}{cc}
1 & \lambda^{1-m} \\
0 & 1
\end{array}\right), m \text { even. }
$$

Theorem 3.1. (SU $(1,1)$ Iwasawa decomposition) [2].

$\Lambda G_{\hat{\sigma}}^{\mathbb{C}}$ is a disjoint union

$$
\Lambda G_{\hat{\sigma}}^{\mathbb{C}}=\mathcal{B}_{1,1} \sqcup \bigsqcup_{n \in \mathbb{Z}^{+}} \mathcal{P}_{n}
$$

where we define the

big cell: $\mathcal{B}_{1,1}:=\Lambda G_{\hat{\sigma}} \cdot \Lambda^{+} G_{\hat{\sigma}}^{\mathbb{C}}$,

n'th small cell: $\mathcal{P}_{n}:=\Lambda S U(1,1)_{\hat{\sigma}} \cdot \omega_{n} \cdot \Lambda^{+} G_{\hat{\sigma}}^{\mathbb{C}}$.

- $\mathcal{B}_{1,1}$, is an open dense subset of $\Lambda G_{\hat{\sigma}}^{\mathbb{C}}$.

- Any $\phi \in \mathcal{B}_{1,1}$ can be expressed as

$$
\phi=F B, \quad F \in \Lambda G_{\hat{\sigma}}, \quad B \in \Lambda^{+} G_{\hat{\sigma}}^{\mathbb{C}},
$$

$F$ is unique up to right multiplication by $G_{\sigma}:=\Lambda G_{\hat{\sigma}} \cap G$.

- The map $\pi: \mathcal{B}_{1,1} \rightarrow \Lambda G_{\hat{\sigma}} / G_{\hat{\sigma}}$ given by $\phi \mapsto[F]$, derived from (5), is a real analytic projection.

3.1.2. The Generalized Weierstrass Representation. For $\lambda_{0} \in \mathbb{S}^{1}$, the SymBobenko formula is given by:

$$
\begin{aligned}
& f^{\lambda_{0}}=-\left.\frac{1}{2 H} \mathcal{S}(F)\right|_{\lambda=\lambda_{0}}, \\
& \mathcal{S}(F):=F i \sigma_{3} F^{-1}+2 i \lambda \partial_{\lambda} F \cdot F^{-1} .
\end{aligned}
$$

We can now state the generalized Weierstrass representation for CMC surfaces in $L^{3}$, which differs from that of the Euclidean case only in that we must restrict to the open set which maps into the big cell $\mathcal{B}_{1,1}$ :

Theorem 3.2. [2] (Holomorphic representation for spacelike CMC surfaces in $L^{3}$ ) Let

$$
\xi=\sum_{i=-1}^{\infty} A_{i} \lambda^{i} \mathrm{~d} z \in \operatorname{Lie}\left(\Lambda G_{\hat{\sigma}}^{\mathbb{C}}\right) \otimes \Omega^{1}(\Sigma)
$$

be a holomorphic 1-form over a simply-connected Riemann surface $\Sigma$, with

$$
a_{-1} \neq 0
$$

on $\Sigma$, where $A_{-1}=\left(\begin{array}{cc}0 & a_{-1} \\ b_{-1} & 0\end{array}\right)$. Let $\phi: \Sigma \rightarrow \Lambda G_{\hat{\sigma}}^{\mathbb{C}}$ be a solution of

$$
\phi^{-1} d \phi=\xi \text {. }
$$

On $\Sigma^{\circ}:=\phi^{-1}\left(\mathcal{B}_{1,1}\right), S U(1,1)-I w a s a w a$ split:

$$
\phi=F B, \quad F \in \Lambda G_{\hat{\sigma}}, \quad B \in \Lambda^{+} G_{\hat{\sigma}}^{\mathbb{C}} .
$$


Then for any $\lambda_{0} \in \mathbb{S}^{1}$, the map $f^{\lambda_{0}}:=\hat{f}^{\lambda_{0}}: \Sigma^{\circ} \rightarrow L^{3}$, given by the SymBobenko formula (6), is a conformal CMC H immersion, and is independent of the choice of $F$ in (7).

\subsection{Examples of the Big Cell Boundary Behaviour.}

3.2.1. Example 1: hyperboloid of two sheets. We start with the holomorphic 1 -form

$$
\xi=\left(\begin{array}{cc}
0 & \lambda^{-1} \\
0 & 0
\end{array}\right) d z, \quad \Sigma=\mathbb{C}
$$

This can be integrated to

$$
\phi=\left(\begin{array}{cc}
1 & z \lambda^{-1} \\
0 & 1
\end{array}\right): \Sigma \rightarrow \Lambda G_{\hat{\sigma}}^{\mathbb{C}}
$$

which takes values in $\mathcal{B}_{1,1}$ for $|z| \neq 1$. An $S U(1,1)$-Iwasawa decomposition is:

$$
\begin{gathered}
\phi=F \cdot B, \quad F: \Sigma \backslash \mathbb{S}^{1} \rightarrow \Lambda G, \quad B: \Sigma \backslash \mathbb{S}^{1} \rightarrow \Lambda^{+} G_{\hat{\sigma}}^{\mathbb{C}}, \\
F=\frac{1}{\sqrt{\varepsilon\left(1-|z|^{2}\right)}}\left(\begin{array}{cc}
\varepsilon & z \lambda^{-1} \\
\varepsilon \bar{z} \lambda & 1
\end{array}\right), \\
B=\frac{1}{\sqrt{\varepsilon\left(1-|z|^{2}\right)}}\left(\begin{array}{cc}
1 & 0 \\
-\varepsilon \bar{z} \lambda & \varepsilon(1-z \bar{z})
\end{array}\right), \quad \varepsilon=\operatorname{sign}\left(1-|z|^{2}\right) .
\end{gathered}
$$

The Sym-Bobenko formula (6) gives

$$
\hat{f}^{1}(z)=\frac{1}{H\left(x^{2}+y^{2}-1\right)} \cdot\left[2 y,-2 x,\left(1+3 x^{2}+3 y^{2}\right) / 2\right],
$$

which is a two-sheeted hyperboloid $\left\{x_{1}^{2}+x_{2}^{2}-\left(x_{0}-\frac{1}{2 H}\right)^{2}=-\frac{1}{H^{2}}\right\}$. Note that the identification of Lorentzian 3 -space $\mathbb{R}^{2,1}$ with $\mathfrak{g}=\mathfrak{s u}_{1,1}$, with inner product given by $\langle X, Y\rangle=\frac{1}{2} \operatorname{trace}(X Y)$, is given by:

$$
e_{1} \leftrightarrow \sigma_{1}, \quad e_{2} \leftrightarrow-\sigma_{2}, \quad e_{3} \leftrightarrow i \sigma_{3} .
$$

Then $\left\langle e_{1}, e_{1}\right\rangle=\left\langle e_{2}, e_{2}\right\rangle=-\left\langle e_{3}, e_{3}\right\rangle=1$.

We can see directly that we are in a small cell precisely when $|z|=1$, because there we have the explicit factorization of $\phi \in \Lambda S U(1,1)_{\hat{\sigma}} \cdot \omega_{2}$. $\Lambda^{+} G_{\hat{\sigma}}^{\mathbb{C}}$ :

$$
\left(\begin{array}{cc}
1 & z \lambda^{-1} \\
0 & 1
\end{array}\right)=\left(\begin{array}{cc}
p \sqrt{z} & \lambda^{-1} q \sqrt{z} \\
\lambda q \sqrt{z}^{-1} & p \sqrt{z}^{-1}
\end{array}\right) \cdot \omega_{2} \cdot\left(\begin{array}{cc}
(p+q) \sqrt{z}^{-1} & 0 \\
-\lambda q \sqrt{z}^{-1} & (p-q) \sqrt{z}
\end{array}\right),
$$

where $p^{2}-q^{2}=1$ and $p, q \in \mathbb{R}$. In other words, $\phi \in \mathcal{P}_{2}$ for $|z|=1$, and we saw above that $\phi \in \mathcal{B}_{1,1}$ otherwise. Note: the surface blows up as $|z| \rightarrow 1$. 
3.2.2. Example 2: numerical experiment.

$$
\xi=\lambda^{-1} \cdot\left(\begin{array}{cc}
0 & 1 \\
100 z & 0
\end{array}\right) d z
$$

Numerically, using Nick Schmitt's program Xlab [6], we do the following:

(1) Integrate with initial condition $\phi(0)=\omega_{1}$, to get $\phi: \Sigma \rightarrow \Lambda G_{\hat{\sigma}}^{\mathbb{C}}$.

(2) Iwasawa split to get $F: \Sigma \rightarrow \Lambda G_{\hat{\sigma}}$.

(3) Compute Sym-Bobenko formula to get $f^{1}: \Sigma \rightarrow L^{3}$.

(4) Use XLab to view the surface.

By construction, we are in the small cell $\mathcal{P}_{1}$ precisely at $z=0$, and at this point we obtain a singularity (Figure 1) which appears to be what is sometimes called a Shcherbak surface singularity [7].

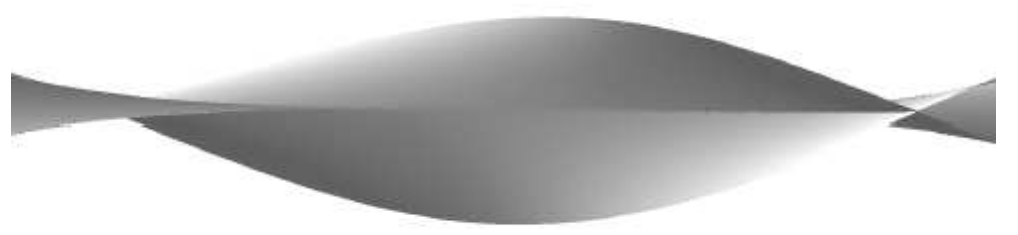

FIgURE 1. The singularity appearing in Example 2

3.3. Results on the Big Cell Boundary Behaviour. In [2] we prove that these examples are, in a certain sense, representative when one encounters the small cells $\mathcal{P}_{1}$ and $\mathcal{P}_{2}$. Specifically:

(1) The map $f^{\lambda_{0}}: \Sigma \rightarrow L^{3}$ always extends to a well defined (and real analytic) map at $z_{0} \in \phi^{-1}\left(\mathcal{P}_{1}\right)$, but is not immersed at such a point.

(2) The map $f^{\lambda_{0}}: \Sigma \rightarrow L^{3}$ always blows up as $z \rightarrow z_{0} \in \phi^{-1}\left(\mathcal{P}_{2}\right)$.

The surface is guaranteed to be smooth provided the holomorphic map $\phi$ takes values in the big cell $\mathcal{B}_{1,1}$. Since the higher order small cells $\mathcal{P}_{i}, i>2$, have higher codimension in the loop group, it is reasonable to expect that generic finite singularities will therefore occur only at $\mathcal{P}_{1}$.

3.4. Applications. It is possible to obtain surfaces with specific geometric properties by choosing the holomorphic 1-form $\xi$ to have a particular special form. One example of an application of this is a classification of CMC surfaces with rotational symmetry in Minkowski space, given in [2]. There are eight natural generic families of such surfaces, and examples from each are shown in Figure 2.

\section{ACKNOWLEDGEMENTS}

In participating in this conference, the first named author is grateful for the support of the Japan Society for the Promotion of Science. 


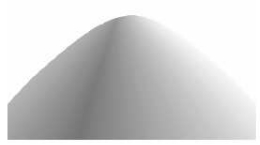

$S_{1 a}\left(\frac{1}{2}, 0\right)$

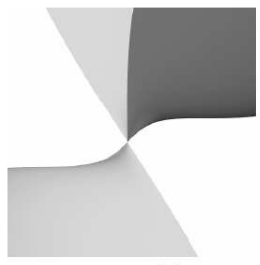

$S_{3}(1, \sqrt{2})$

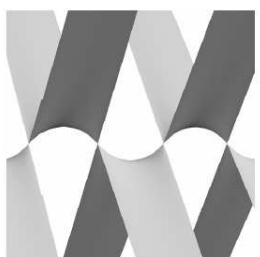

$S_{1 b}(2,0)$

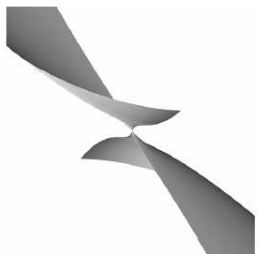

$T_{1}(1,4)$

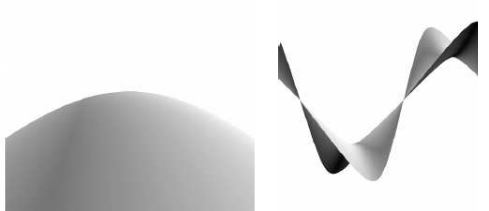

$S_{2 a}\left(-\frac{1}{2}, 0\right)$

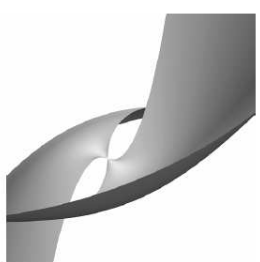

$T_{2}(-1,4)$
$S_{2 b}(-2,0)$

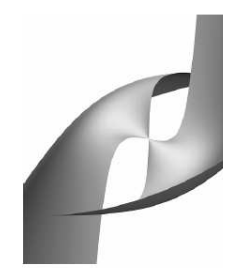

$T_{3}(-1, \sqrt{2})$

FiguRE 2. Examples from each of the eight families of surfaces with rotational symmetry in $L^{3}$. Images made by $X L a b$ $[6]$.

\section{REFERENCES}

[1] D Brander and J Dorfmeister, Generalized DPW method and an application to isometric immersions of space forms, Math. Z. (2009), DOI: 10.1007/s00209-008-0367-9.

[2] D Brander, N Schmitt, and W Rossman, Holomorphic representation of constant mean curvature surfaces in Minkowski space: consequences of non-compactness in loop group methods, arXiv:math/0804.1596 (2008).

[3] J Dorfmeister, F Pedit, and $\mathrm{H}$ Wu, Weierstrass type representation of harmonic maps into symmetric spaces, Comm. Anal. Geom. 6 (1998), 633-668.

[4] I M Krichever, An analogue of the d'Alembert formula for the equations of a principal chiral field and the sine-Gordon equation, Dokl. Akad. Nauk SSSR 253 (1980), no. 2, 288-292.

[5] A Pressley and G Segal, Loop groups, Oxford Math. Monographs, Clarendon Press, Oxford, 1986.

[6] N Schmitt, XLab, software, www.mathematik.unituebingen.de/ab/DifferentialGeometrie.

[7] O Shcherbak, Wave fronts and reflection groups, Uspekhi Mat. Nauk 43 (1988), no. 3(261), 125-160. Translation in: Russian Math. Surveys 43 (1988), no. 3, 149-194.

Department of Mathematics, Technical University of Denmark, MatematikTORVET, B. 303 S, Lyngby, 2800, Denmark

E-mail address: brander@math.kobe-u.ac.jp

Department of Mathematics, Faculty of Science, Kobe University, Japan

E-mail address: wayne@math.kobe-u.ac.jp

GeometrieWerkstatt, Mathematisches Institut, Universität Tübingen, GerMANY

E-mail address: nschmitt@mathematik.uni-tuebingen.de 